\title{
HYDROGEL CHECK VALVE WITH NON-ZERO CRACKING PRESSURE FOR USE AS A POTENTIAL ALTERNATIVE HYDROCEPHALUS TREATMENT METHOD
}

\author{
H.N. Schwerdt ${ }^{*}$, R. Bristol ${ }^{2}$, and J. Chae ${ }^{l}$ \\ ${ }^{1}$ Arizona State University, Tempe, Arizona, USA \\ ${ }^{2}$ Phoenix Children's Hospital, Phoenix, Arizona, USA
}

\begin{abstract}
An alternative method of treating hydrocephalus by means of cranially confining and miniaturizing draining mechanisms may offer greater safety and reliability than standard cerebrospinal fluid (CSF) shunts that suffer from high failure rates partly related to their physically large form (2 long catheters and 1 valve) and implant coverage (brain to distal organs). This passive check valve targets restoration of near natural CSF draining operations and attempts to mitigate common valve complications such as back flow and steady state leakage. A simple perforated hydrogel membrane forms the basic check valve structure and its swelling characteristics provide the sealing to diminish leakage. Preliminary measurements demonstrate its ability to operate within an appropriate range of $-800<\Delta P<600 \mathrm{mmH}_{2} \mathrm{O}$, establish a targeted $P_{T} \approx 20-110 \mathrm{mmH}_{2} \mathrm{O}$, and substantially lower reverse flow leakage for $\triangle P<P_{T}$
\end{abstract}

\section{INTRODUCTION Background}

Hydrocephalus, a chronic neurological and, in most cases, congenital disorder, occurs due to excessive accumulation of CSF within the ventricles of the brain causing fatally high increases in intracranial pressures (ICP) [1-3]. The disorder may lead to abnormal enlargement of the head, convulsions, mental disabilities, and even death. Most forms of this condition are thought to originate due to faulty arachnoid granulations that serve as natural valve formations protruding from the meningeal membrane, arachnoid mater, surrounding the brain and that normally direct CSF from the subarachnoid space (SAS) of the brain to the sagittal sinus immediately exterior to the meninges and enclosed within the cranium [2]. Current methods to treat hydrocephalus involve, primarily, implanting a macroscopic valve (CSF shunt) into the brain to divert the accumulating CSF through an intracranial and subcutaneous long tube (catheter) outside of the cranium and to the abdominal cavity or other distal organs $[1,3,4]$ (Fig. 1). However, more than $50 \%$ of these shunts fail within the first two years of implantation, instigating additional invasive surgeries [3]. Some of these complications may be attributed to the shunts' physically large and lengthy form making them susceptible to external forces and/or siphoning effects. Additionally, intracranial catheters potentially expose the brain and increase risks of infection.

\section{Current Technology}

Normally closed MEMS check valves have been studied for over 20 years, and target mainly 3 operating parameters [5-9]: 1) high diodicity or unidirectionality of flow $\left.\left(Q_{O} \geq 0\right), 2\right)$ zero leakage when applied differential pressure $(\triangle P)$ is less than the cracking pressure $\left(P_{T}\right)$, and 3$)$ a designated non-zero or zero $P_{T}$ (the former is targeted for CSF draining). However, existing work usually reveals deficiencies in one or more of these targeted properties, and especially so in avoiding reverse flow leakage that occurs due to inadequate sealing of the valve when $\Delta P<P_{T}[5,8,9]$. Other limitations may involve reverse flow leakage at higher back pressures $(\triangle P<<0)$ [5], permanent deformation that directly diminishes repeatability [8], stiction of the valve seat that affects reproducibility and yield, and/or finite leakage observed after longer or multiple periods of operation undermining its utility in permanent chronic implants. To reduce many of these failure mechanisms, more complex valve designs and fabrication processes have been developed to realize adequate sealing with low or virtually zero static and reverse flow leakage and higher reproducibility and durability [10-11]. These more complex valves were formed on PDMS and were constructed for application in drug delivery [10] or regulating intraocular pressure (IOP) [11] for glaucoma treatment and demonstrated effective operation as implemented in animal models. Unlike drainage devices used in treating glaucoma where $Q_{O} \approx 1-5 \mu \mathrm{L} / \mathrm{min}$ and $P_{T} \approx 68-203 \mathrm{mmH}_{2} \mathrm{O}$, CSF draining for hydrocephalus involves substantially higher flow rates $\left(Q_{O} \approx 0.1-3.0 \mathrm{~mL} / \mathrm{min}\right)$ and may target more variable cracking pressures $\left(P_{T} \approx 10-230 \mathrm{mmH}_{2} \mathrm{O}\right)$ [4]. "Arachnoid mimicking" microvalve arrays using parylene/PDMS composite flap valves were recently developed for application in hydrocephalus, but retained challenges in leakage and diodicity [5].

\section{Current HCP Treatment}

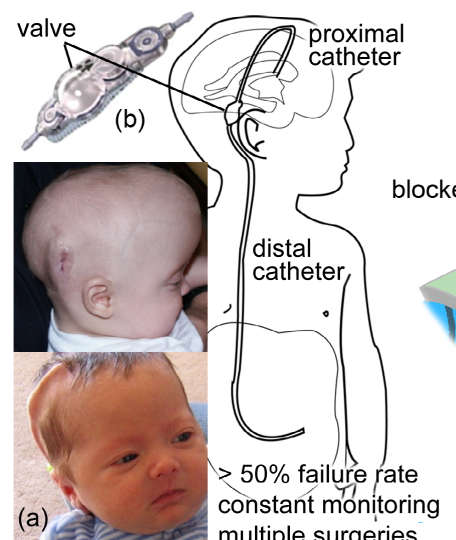

Alternative Approach

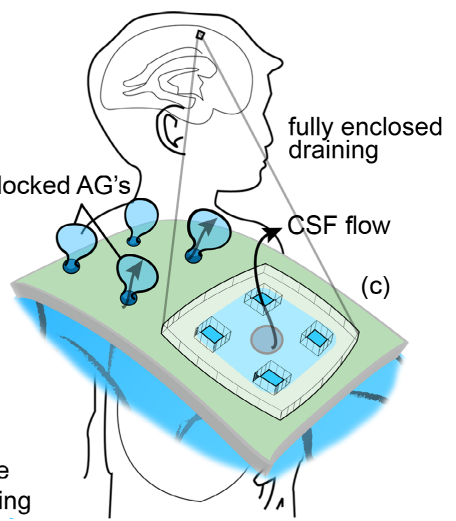

Figure 1: Depiction of current hydrocephalus (HCP) treatment methods versus a fully enclosed approach that would eliminate the need for relatively large subcutaneous catheters and valves. (a) Photographs of infants with HCP demonstrating noticeable shunt protruding the skin as well as enlargement of the head. (b) Photograph of commercial macroscopic shunt valve used to regulate CSF flow through connected catheters (Medtronic, Inc.). (c) Illustration of how HCP affects normal CSF draining through malfunctioning blocked arachnoid granulations (AGs) and how a microvalve may be appended to direct CSF flow from the brain at the associated meningeal membrane without requiring excess through-cranium catheters or distal tubing.

\section{Novelty and Impact}

The check valve presented herein aims to alleviate challenges in valve sealing and finite leakage by utilizing the intrinsic swelling behavior of hydrogels. Hydrogel polymers swell and expand when immersed in liquids that diffuse through microscopic pores in the gel matrix. This swelling phenomenon forms the basic passive check valve functionality and may effectively provide secure sealing between the valve seat and inlet to minimize static and reverse flow leakage issues. Hydrogel materials have been 
employed in valves to create environmentally (ie. $\mathrm{pH}$ or temperature) responsive valves for microfluidic lab-on-a-chip applications for in-plane operation [9]. Unlike previously reported hydrogel valves, the valve of this work is composed of only silicon and hydrogel and is fabricated using planar micromachining techniques for out-of-plane operation. As a potential hydrocephalus treatment method, this valve may be concealed subcranially and effectively substitute CSF draining operations of the obstructed arachnoid granulations (Fig. 2). Subsequent sections of this paper will delineate the passive check valve's design and fundamental operation, fabrication process, and measurement results.

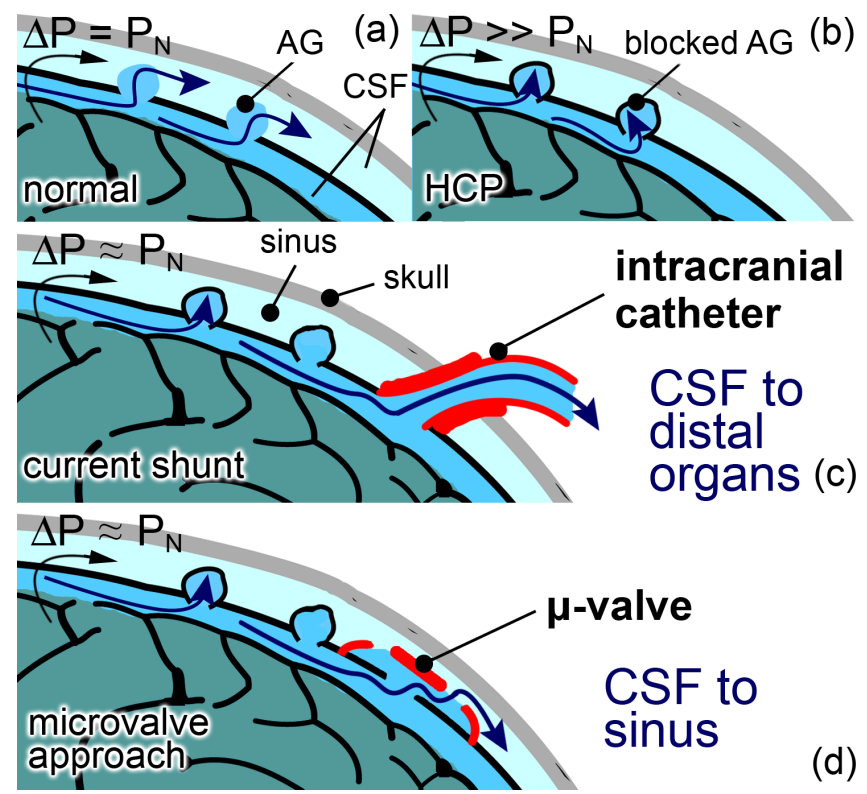

Figure 2: Illustration of arachnoid granulation function as a oneway valve (protruding structures from the dura mater) to direct CSF flow from the subarachnoid space (SAS) enclosing the brain to the sagittal sinus (top right). (a) Under normal conditions the intracranial differential pressure (ICP) is around $-100<\Delta P<350$ $\mathrm{mmH}_{2} \mathrm{O}$ ( $P_{\mathrm{N}}$, in figure, indicates this normal ICP range). (b) With hydrocephalus $\triangle P$ may reach fatally higher levels due to buildup of CSF within the brain. (c) Current shunts direct CSF from the brain to the stomach or other distal organs, requiring throughcranium catheters. (d) The hydrogel microvalve attempts to alleviate this perilous condition to substitute CSF draining operations of blocked arachnoid granulations (AGs) and diverting CSF directly to the sinus, as in normal conditions.

\section{MATERIALS AND METHODS}

\section{Operation}

The check valve essentially functions to provide a normally closed one way conduit for fluid flow and is in the form of a single suspended perforated square diaphragm made of hydrogel anchored to a silicon substrate (Fig. 3). Both valve structures, hydrogel and silicon, are approved biocompatible materials $[12,13]$. In its closed state $\left(\triangle P<P_{T}\right)$, the water induced expansion of the hydrogel seals the inlet port and prevents fluid outflow. At $\triangle P \geq P_{T}$, sufficient inlet pressure overwhelms hydrogel swelling forces allowing the valve to open, at which point the opened valve's fluid flow rate $\left(Q_{O}\right)$ is governed by the level of displacement of the hydrogel membrane. The valve's hydrogel material properties and dimensions determine the operating range of $Q_{O}$ along with $\Delta P$, as well as $P_{T}$.
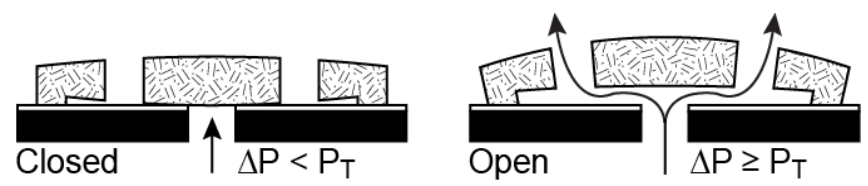

Figure 3: Designated closed and open states of the hydrogel microvalve. In the closed state (left) the hydrogel's swelling force allows the perforated membrane to seal the inlet channel and block flow (when $\triangle P<P_{T}$, cracking pressure). In the open state, when ICP reaches $P_{T}\left(\triangle P \geq P_{T}\right)$, the membrane deflects upwards to allow fluid to flow through the perforations through the membrane.

\section{Design}

The check valve targets operation at $Q_{O} \approx 0.1-3.0 \mathrm{~mL} / \mathrm{min}$, $200<\Delta P<600 \mathrm{mmH}_{2} \mathrm{O}$, and $P_{T} \approx 10-230 \mathrm{mmH}_{2} \mathrm{O}$ as pertains to observed CSF draining in current hydrocephalus shunts and normal and increased ICP levels found in hydrocephalus patients $[1,3,4]$. A perforated square diaphragm performs basic valve operations by deflecting and allowing fluid to flow through exposed perforations in response to the pressure $(\Delta P)$ across the valve. The deflection of a suspended square membrane in response to pressure may be estimated by [14],

$$
w_{0}=0.802 b \cdot\left(\frac{P b}{E t}\right)^{1 / 3}
$$

where $w_{0}$ is maximum center displacement, $b$ is membrane side length halved, $P$ is pressure, $E$ is Young's modulus $(E \approx 100-300$ $\mathrm{kPa}$ for hydrogels) [15], and $t$ is membrane thickness. Volumetric fluid flow rate $\left(Q_{O}\right)$ through the valve may then be modeled by a linear expression, $Q_{O}=\Delta P / R_{h}$, where $R_{h}$ is hydraulic resistance, which for a rectangular orifice may be expressed as,

$$
R_{h} \approx \frac{12 \mu L}{w h^{3}(1-0.603 h / w)}
$$

where $\mu$ is dynamic viscosity of the fluid ( $\mu($ water $) \approx 0.68$ at $38^{\circ} \mathrm{C}$ ), $L$ is channel length, $w$ is channel width, and $h$ is channel height. $R_{h}$ may be modeled as a series configuration of resistors correlated with the inlet channel of the silicon substrate, the variable gap formed by deflection of the hydrogel membrane, and the perforation outlet vias of the membrane. Based on this simplified model and the intended pressure range $(\Delta P)$ and targeted flow rates $\left(Q_{O}\right)$, various dimensions for the check valve are selected in the range of $b=600-1150 \mu \mathrm{m}, t \approx 100-260 \mu \mathrm{m}$, inlet channel diameter of 400-700 $\mu \mathrm{m}$, and perforation outlet diameters of 200-400 $\mu \mathrm{m}$.

Along with its physical dimensions ( $b$ and $t$ ), flow characteristics of the passive membrane valve are predominantly governed by the hydrogel material properties that will determine $E$ in (1) as well as $P_{T}$. According to Flory theory and its extensions [16], the volumetric swelling ratio for a highly swollen gel network may be estimated by

$$
\left(\frac{V}{V_{0}}\right)^{-2 / 3}=\frac{k}{\zeta Z}
$$

where $V$ and $V_{0}$ are swollen and dry gel volumes, respectively, $k$ is gel's elasticity, $\zeta$ is degree of gel ionization, and $Z$ is potential ions per dry gel volume. Flory theory also suggests a linear relationship between $k$ and the ratio of crosslinker, which may further indicate that the hydrogel volume is to the $(-2 / 3)$ power proportional to crosslinker ratio. This swelling dependence may be used to predict $P_{T}$ of the valve. The hydrogel membrane remains swollen as it is immersed in liquid at all times. 


\section{Fabrication}

The fabrication process is outlined in Fig. 4 and involves i) patterning and hardbaking $4 \mu \mathrm{m}$ thick photoresist (AZ P4330-RS, AZ Electronic Materials) sacrificial layers on $0.2 \mu \mathrm{m}$ plasma enhanced chemical vapor deposited (PECVD) $\mathrm{SiO}_{2} 500 \mu \mathrm{m}$ thick silicon $<100>$ substrates followed by adhesion promotion, ii) application and UV exposure patterning of hydrogel solution confined by $150 \mu \mathrm{m}$ rubber spacers between mask and substrate, iii) deep reactive ion etching (DRIE) of backside inlet channels through silicon, followed by wet etching of topside exposed $\mathrm{SiO}_{2}$ and photoresist, and iv) immersion in water to swell hydrogels. Completed valves are shown in Fig. 5. The chemical composition of the hydrogel solution comprises the monomer, 2-hydroxyethyl methacrylate (HEMA), crosslinker, ethylene glycol dimethacrylate (EGDA), and photoinitiator, 2,2-dimethoxy-2-phenylacetophenone (DMPA), at a volumetric ratio of 1.0/0.04/0.1.

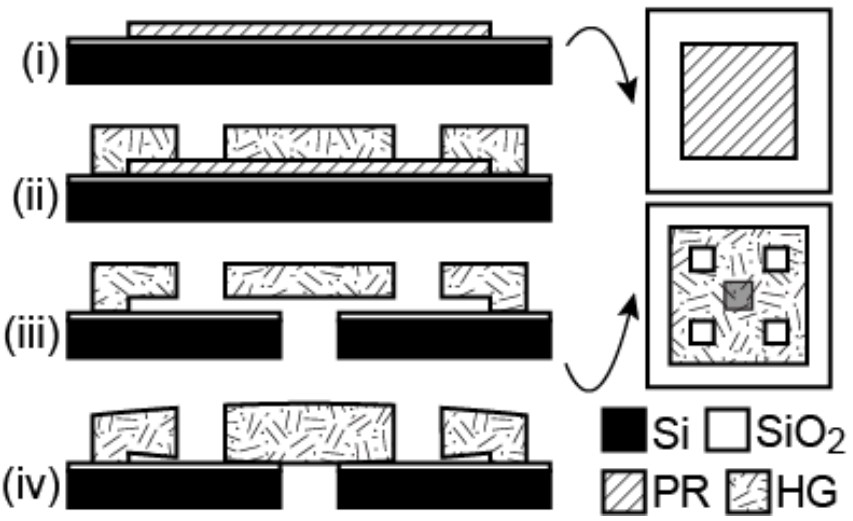

Figure 4: Cross-sectional (left) and top-view (right) fabrication process flow diagrams. (i) $4 \mu \mathrm{m}$ thick sacrificial photoresist (PR) is spun and patterned on a silicon wafer, coated with $0.2 \mu \mathrm{m}$ thick PECVD SiO ${ }_{2}$, followed by overnight treatment in an adhesion promoter. (ii) $150 \mu \mathrm{m}$ thick rubber spacers are fixed to the perimeter of the wafer and hydrogel (HG) solution is poured into the enclosed area, and patterned by UV. Uncured HG is rinsed away with isopropyl alcohol. (iii) Backside channels through silicon are etched using DRIE to expose topside $\mathrm{SiO}_{2}$. Remaining $\mathrm{SiO}_{2}$ and sacrificial $\mathrm{PR}$ are etched away using buffered hydrofluoric acid and acetone/isopropyl alcohol, respectively. (iv) Fabricated hydrogel microvalves are placed in water to acquire their swollen state and seal the channels in their initial closed state.

\section{RESULTS}

\section{Measurements}

Benchtop testing of the hydrogel check valve was performed using continuous syringe pump injection of water to generate $\Delta P$ across the valve and recording output steady state $Q_{O}$ (Fig. 6). Hydrogel membrane thicknesses $(t)$ ranged from 110 to $260 \mu \mathrm{m}$ and $b$ from 0.6 to $1.15 \mathrm{~mm}$. The intended operating fluid, CSF, has a density of $1.003-1.008 \mathrm{~g} / \mathrm{cm}^{3}$ and $\mathrm{pH}$ of 7.4 [2], which closely match those of water and may validate its use as the working fluid for benchtop testing. $\Delta P$ across the valve was acquired by converting voltage output from a differential pressure transducer (PX26-001DV, Omega). $Q_{O}$ was monitored by measuring float height through a rotameter (FL-1441-G, Omega) with 5\% accuracy and $\sim 3.5 \mu \mathrm{L} / \mathrm{min}$ resolution at discrete intervals (15 to 45 minutes) upon stabilization of $\Delta P$.

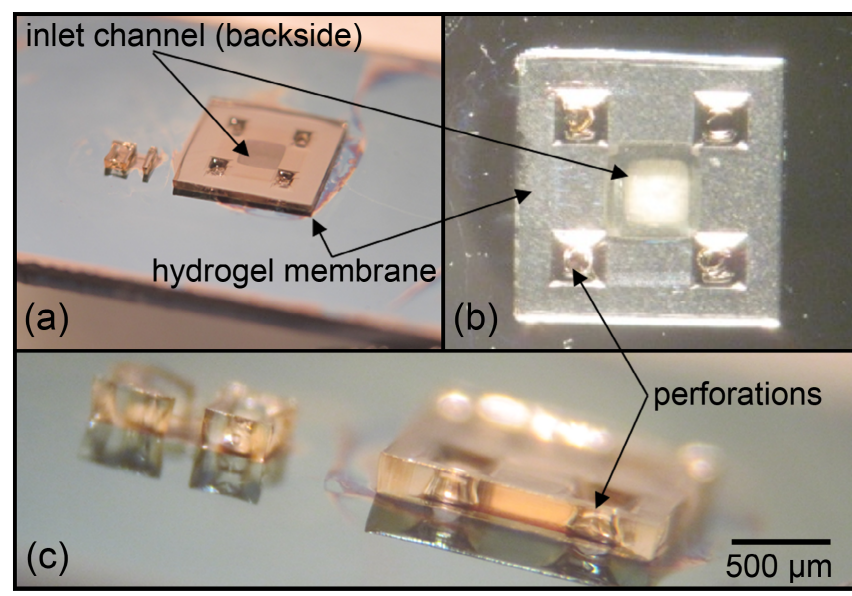

Figure 5: Photographs of fabricated hydrogel microvalves. (a) Angled die level view. (b) Top view of hydrogel microvalve. (c) Cross-sectional view of microvalve with scale bar.

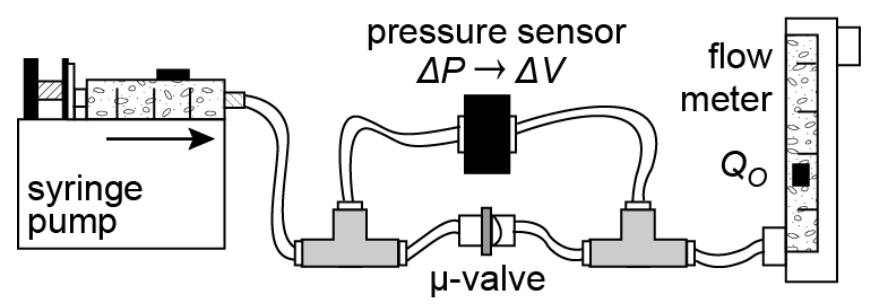

Figure 6: Experimental test setup. A syringe pump (left) manually generates pressure across the $\mu$-valve (bottom center). A pressure sensor (top center) measures differential pressure $(\Delta P)$ across the valve through its voltage output $(\Delta V)$. A flow meter (right) measures the output flow rate of the microvalve $\left(Q_{O}\right)$.

\section{Discussion}

Fluid flow response for several different hydrogel membrane geometries are shown in Fig. 7 and demonstrate high diodicity with no observable static or reverse flow leakage and targeted $P_{T} \approx 20-110 \mathrm{mmH}_{2} \mathrm{O}$. The valves are capable of tolerating high back pressures $\left(\triangle P<-800 \mathrm{mmH}_{2} \mathrm{O}\right)$ without noticeable deformation or degradation while maintaining desired $P_{T}$. Fig. 8 shows preliminary over time measurements demonstrating the valve's ability to retain targeted $P_{T}$ with a variability of $\pm 7.5 \mathrm{mmH}_{2} \mathrm{O}$. Table 1 summarizes the measured results of the valves along with comparison to targeted specifications.

The maximum $Q_{O}$ was slightly under $0.20 \mathrm{~mL} / \mathrm{min}$ at the maximum $\triangle P=600 \mathrm{mmH}_{2} \mathrm{O}$. Depending on the treatment requirements for hydrocephalus, higher flow rates may be needed signifying lowering the hydraulic resistance, $R_{h}$, of the microvalve. This may be implemented indirectly by simply utilizing a parallel array of multiple valves. Changes in hydrogel membrane geometry and dimensions may also be made to decrease $R_{h}$ as well as optimize other flow parameters as required for the specific treatment protocol.

The fabrication process revealed several challenges in yield and reproducibility mainly owing to stiction and photoresist release. Nonetheless, slight optimizations by increasing the thickness of the sacrificial photoresist layer and/or treating or coating hydrogel or $\mathrm{SiO}_{2}$ surfaces may alleviate these challenges. 


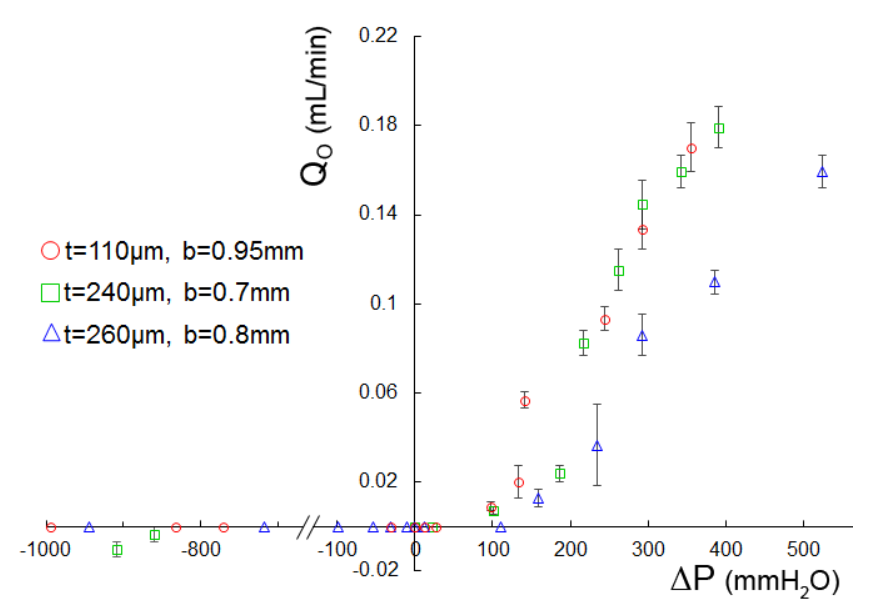

Figure 7: Measured flow response of hydrogel microvalves demonstrating $P_{T} \approx 20-110 \mathrm{mmH}_{2} \mathrm{O}$ that is within targeted $P_{T} \approx 10$ $230 \mathrm{mmH}_{2} \mathrm{O}$ with static and reverse flow sealing and high diodicity where $Q_{O}=0$ at steady state $\left(\triangle P=0 \mathrm{mmH}_{2} \mathrm{O}\right)$ and at high back pressures $\left(\triangle \mathrm{P}<-800 \mathrm{mmH}_{2} \mathrm{O}\right)$.

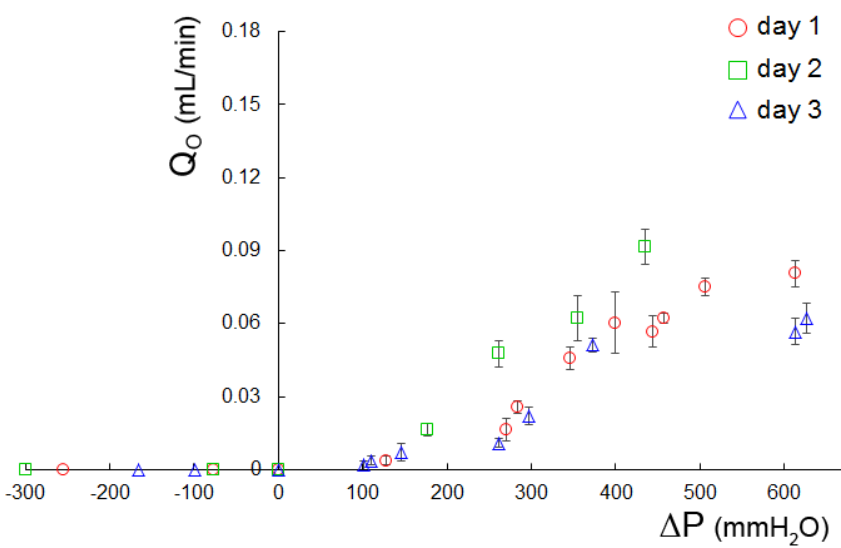

Figure 8: Preliminary over time measurements of a single hydrogel microvalve device depicting low variability of $P_{T}$.

\section{CONCLUSION}

A hydrogel microvalve operating at targeted $\triangle P$ and $Q_{O}$ levels suitable for CSF draining with negligible or zero observable leakage may strengthen its potential utility as an alternative hydrocephalus treatment method. The valve is relatively simple in design and only comprises two materials, silicon and hydrogel, both of which are biocompatible. Benchtop testing to characterize its long term performance and durability are ongoing. Furthermore, additional tests may be performed to characterize valve fluid flow properties as relates to its geometry and hydrogel material composition to confirm manual controllability of $P_{T}$ as well as other flow parameters (ie. hydraulic resistance, $R_{h}$ ). Future work to ensure its reliability and durability as a permanent medical implant will entail long term and in vivo testing.

Table 1: Targeted and measured specifications of hydrogel check valve.

\begin{tabular}{lcc}
\hline \hline & Targeted & Measured \\
\hline$\Delta P$ & $-200-600 \mathrm{mmH}_{2} \mathrm{O}$ & $-800-600 \mathrm{mmH}_{2} \mathrm{O}$ \\
$Q_{O}$ & $0.1-3.0 \mathrm{~mL} / \mathrm{min}$ & $0.01-0.20 \mathrm{~mL} / \mathrm{min}$ \\
$P_{T}$ & $10-230 \mathrm{mmH}_{2} \mathrm{O}$ & $20-110 \mathrm{mmH}_{2} \mathrm{O}$ \\
\hline \hline
\end{tabular}

\section{ACKNOWLEDGMENT}

This project was partially supported by the Arizona State University Graduate and Professional Student Association's JumpStart Grant. Travel support has been generously provided by the Transducer Research Foundation.

\section{REFERENCES}

[1] A. Chabrerie and P.M. Black, "Ventricular Shunts," Journal of Intensive Care Medicine, 15, 218 (2002).

[2] A. Brodbelt and M. Stoodley, "CSF Pathways: A Review," British Journal of Neurosurgery, 21, 510 (2007).

[3] J.M. Drake, J.R.W. Kestle, and S. Tuli, "CSF Shunts 50 Years On-Past, Present, and Future," Children's Nervous System, 16,800 (2000).

[4] M. Czosnyka, Z. Czosnyka, H. Whitehouse, and J.D. Pickard, "Hydrodynamic Properties of Hydrocephalus Shunts: United Kingdom Shunt Evaluation Laboratory," Journal of Neurology, Neurosurgery, and Psychiatry 62, 43 (1997).

[5] J. Oh, G. Kim, F. Kralick, and H. Noh, "Design and Fabrication of a PDMS/Parylene Microvalve for the Treatment of Hydrocephalus," Journal of Microelectromechanical Systems, 20, 811 (2011).

[6] K. Oh and C. Ahn, "A Review of Microvalves," Journal of Micromechanics and Microengineering, 16, R13 (2006).

[7] P. Chen, D. Rodger, E. Meng, M. Humayun, and Y. Tai, "Surface-Micromachined Parylene Dual Valves for On-Chip Unpowered Microflow Regulation," Journal of Microelectromechanical Systems, 16, 223 (2007).

[8] J. Tirén, L. Tenerz, and B. Hok, "A Batch-Fabricated NonReverse Valve with Cantilever Beam Manufactured by Micromachining of Silicon," Sensors and Actuators, 18, 389 (1989).

[9] D. Kim and D. Beebe, "A Bi-Polymer Micro One-Way Valve," Sensors and Actuators, 136, 426 (2007).

[10] R. Lo, P. Li, S. Saati, R.N. Agrawal, M.S. Humayan, and E. Meng, "A Passive MEMS Drug Delivery Pump for Treatment of Ocular Diseases," Biomedical Microdevices, 11, 959 (2009).

[11] S. Moon, S. Im, J. An, C.J. Park, H.G. Kim, S.W. Park, H.I. Kim, and J. Lee, "Selectively Bonded Polymeric Glaucoma Drainage Device for Reliable Regulation of Intraocular Pressure," Biomedical Microdevices, 10.1007/s10544-0119609-4 (2011).

[12] J. Montheard, M. Chatzopoulos, and D. Chappard, "2Hydroxyethyl Methacrylate (HEMA): Chemical Properties and Applications in Biomedical Fields," Journal of Macromolecular Science Part C, 32, 1 (1992).

[13] G. Kotzar, M. Freas, P. Abel, A. Fleischman, S. Roy, C. Zorman, J.M. Moran, and J. Melzak, "Evaluation of MEMS Materials of Construction for Implantable Medical Devices," Biomaterials, 23, 2737 (2002).

[14] S. Timoschenko, Theory of Plates and Shells, McGraw-Hill, New York, 1940.

[15] H. Li, T.Y. Ng, Y.K. Yew, and K.Y. Lam, "Modeling and Simulation of the Swelling Behavior of $\mathrm{pH}$-stimulusResponsive Hydrogels," Biomacromolecules, 6, 109 (2005).

[16] S.H. Gehrke, G.P. Andrews, and E.L. Cussler, "Chemical Aspects of Gel Extraction," Chemical Engineering Science, 41, 2153 (1986).

\section{CONTACT}

*H.N. Schwerdt; hschwerd@asu.edu 